\title{
Acute surgical management of children with ruptured brain arteriovenous malformation
}

\author{
*Sarah Stricker, MD, ${ }^{1}$ Grégoire Boulouis, MD, 2,3 Sandro Benichi, MD, 1,2 Marie Bourgeois, MD,1 \\ Florent Gariel, MD, ${ }^{3}$ Lorenzo Garzelli, MD, ${ }^{3}$ Jean-François Hak, MD, ${ }^{1,2}$ Quentin Alias, MD, ${ }^{1,2}$ \\ Basile Kerleroux, MD, ,,3 Kevin Beccaria, MD, PhD, ${ }^{1,2}$ Anaïs Chivet, MD,1,2 \\ Timothée de Saint Denis, MD, ${ }^{1}$ Syril James, MD, ${ }^{1}$ Giovanna Paternoster, MD, ${ }^{1}$ \\ Michel Zerah, MD, PhD, 1,2 Manoelle Kossorotoff, MD, PhD, 1,2 Nathalie Boddaert, MD, PhD, 1,2,4 \\ Francis Brunelle, MD, PhD, ${ }^{1,2}$ Philippe Meyer, MD, ${ }^{1}$ Stéphanie Puget, MD, PhD, \\ Olivier Naggara, MD, PhD, ${ }^{1-3}$ and Thomas Blauwblomme, MD, PhD ${ }^{1,2,4}$
}

\begin{abstract}
${ }^{1}$ APHP, Necker Hospital; 2Université de Paris; ${ }^{3}$ NSSERM U1266, Department of Neuroradiology, Sainte-Anne Hospital, GHU Paris Psychiatry and Neurosciences; and ${ }^{4}$ INSERM U1163, Imagine Institute, Paris, France

OBJECTIVE Rupture of brain arteriovenous malformation (AVM) is the main etiology of intracerebral hemorrhage $(\mathrm{ICH})$ in children. Ensuing intracranial hypertension is among the modifiable prognosis factors and sometimes requires emergency hemorrhage evacuation $(\mathrm{HE})$. The authors aimed to analyze variables associated with $\mathrm{HE}$ in children with ruptured AVM.
\end{abstract}
METHODS This study was a single-center retrospective analysis of children treated for ruptured AVM. The authors evaluated the occurrence of $\mathrm{HE}$, its association with other acute surgical procedures (e.g., nidal excision, decompressive hemicraniectomy), and clinical outcome. Variables associated with each intervention were analyzed using univariable and multivariable models. Clinical outcome was assessed at 18 months using the ordinal King's Outcome Scale for Childhood Head Injury.

RESULTS A total of 104 patients were treated for 112 episodes of ruptured AVM between 2002 and 2018. In the 51 children ( $45.5 \%$ of cases) who underwent HE, 37 procedures were performed early (i.e., within 24 hours after initial cerebral imaging) and 14 late. Determinants of HE were a lower initial Glasgow Coma Scale score (adjusted odds ratio [aOR] 0.83, 95\% Cl 0.71-0.97 per point increase); higher $\mathrm{ICH} /$ brain volume ratio (aOR 18.6, 95\% Cl 13-26.5 per percent increase); superficial AVM location; and the presence of a brain herniation (aOR 3.7, 95\% Cl 1.3-10.4). Concurrent nidal surgery was acutely performed in $69 \%$ of Spetzler-Martin grade I-II ruptured AVMs and in $25 \%$ of Spetzler-Martin grade III lesions. Factors associated with nidal surgery were superficial AVMs, late HE, and absent alteration of consciousness at presentation. Only 8 cases required additional surgery due to intracranial hypertension. At 18 months, overall mortality was less than $4 \%$, $58 \%$ of patients had a favorable outcome regardless of surgical intervention, and $87 \%$ were functioning independently.

CONCLUSIONS HE is a lifesaving procedure performed in approximately half of the children who suffer AVM rupture. The good overall outcome justifies intensive initial management. https://thejns.org/doi/abs/10.3171/2020.8.PEDS20479

KEYWORDS arteriovenous malformation; intracerebral hemorrhage; hematoma evacuation; nidal surgery; vascular disorders

$\mathrm{P}$ EDIATRIC brain arteriovenous malformations (AVMs) are the main cause for intracerebral hemorrhage (ICH) in children., ${ }^{1,2}$ Ruptured AVM-related ICH may cause cerebral damage and result in increased intracranial pressure (ICP), leading to brain herniation, sei- zures, neurological deficits, altered consciousness, and eventually death if not properly treated at the acute phase.

Along with critical care management, emergency surgery plays a preeminent role in reducing ICP, secondary brain injuries due to mass effect of ICH, and early re-

ABBREVIATIONS aOR = adjusted odds ratio; $A V M=$ arteriovenous malformation; $D C=$ decompressive hemicraniectomy; EVD = external ventricular drain; GCS = Glasgow Coma Scale; HE = hemorrhage evacuation; ICH = intracerebral hemorrhage; ICP = intracranial pressure; IVH = intraventricular hemorrhage; KOSCHI = King's Outcome Scale for Childhood Head Injury; TBV = total brain volume.

SUBMITTED June 2, 2020. ACCEPTED August 3, 2020.

INCLUDE WHEN CITING Published online January 22, 2021; DOI: 10.3171/2020.8.PEDS20479.

* S.S. and G.B. share first authorship of this work. O.N. and T.B. share senior authorship of this work. 
bleeding. Acute surgical interventions include external ventricular drain (EVD) insertion, hemorrhage evacuation (HE), targeted AVM nidus excision, and, rarely, decompressive hemicraniectomy (DC). ${ }^{3-5}$ Yet, given the scarcity of the literature and the rarity of the condition, there are no surgical guidelines-hence, acute surgical decisions are mainly extrapolated from traumatic brain injury cases, from adults, and from local institutional experience.

In a large pediatric cohort of ruptured AVMs, we aimed to retrospectively analyze the determinants of surgical management at the acute phase, with an emphasis on $\mathrm{HE}$, and their association with clinical outcome.

\section{Methods}

\section{Study Design, Data Extraction, and Clinical Data}

The manuscript was prepared in accordance with the Strengthening the Reporting of Observational Studies in Epidemiology (STROBE) statement. ${ }^{6}$ Data were retrospectively queried from a previously described prospective registry of children (age $<18$ years) presenting with ICH at the pediatric hospital (Necker-Enfants Malades, Paris, France). ${ }^{2}$ Children with AVM rupture between 2002 and 2018 were included in the current study.

As is customary for observational studies with retrospective analysis of routinely acquired data, generalized patient consent was used, written informed consent was waived, and patients or the children's parents could oppose the use of their health-related data for the purpose of research. Study protocol was approved by the institutional review board. Retrospective data collection was completed by studying the patients' medical charts with the use of a local data warehouse. ${ }^{7}$ Clinical data retrieved included age at event; sex; initial Glasgow Coma Scale (GCS) score; presence of seizures; and clinical signs of elevated ICP; as well as neurological deficits (namely motor deficits, visual field deficits, aphasia, and cerebellar syndrome). Altered mental status was considered for initial GCS score $<9$. Clinical outcome, assessed 18 months after ICH, was defined as favorable if the King's Outcome Scale for Childhood Head Injury (KOSCHI) score was $\geq 5 \mathrm{~A}$, as unfavorable outcome with mild disabilities for scores 4A and 4B, and as unfavorable with severe disabilities or vegetative state for KOSCHI scores 3 and $2 .{ }^{8}$

\section{Imaging Analysis}

The following variables were retrieved from acute imaging sequences: ICH location, ICH volume (in $\mathrm{ml}$ ) and its ratio to the measured total brain volume (TBV; in $\mathrm{ml}$ ) (ICH/TBV), and hydrocephalus (according to ventricular dilatation of the temporal horns with a diameter $>5 \mathrm{~mm}){ }^{9}$ The extent of the intraventricular hemorrhage (IVH) was semiquantitatively assessed using the modified Graeb score (ranging from 0 to 32), which is based on the distribution and amount of IVH and associated ventricular dilatation. ${ }^{10}$ Three types of brain herniation were distinguished: herniation of the falx, herniation of the temporal lobes, and herniation of the cerebellar tonsils. Angioarchitectural AVM characteristics included Spetzler-Martin grade, eloquent location, associated aneurysms, any deep venous drainage, and deep AVM localization. In order to strengthen the outcome of statistical analysis given the size of our cohort, AVMs were grouped according to the classification described by Spetzler and Ponce into class A (Spetzler-Martin grade I and II), class B (Spetzler-Martin grade III), and class C (Spetzler-Martin grade IV and V). ${ }^{11}$ Hemorrhages reaching the cortex or located immediately subcortically were defined as superficial.

\section{Treatment Modalities}

Because our hospital is the on-call pediatric neurosurgical department for Île-de-France region (population 12 million), children can be referred from other hospitals in case of ICH or directly from home in case of altered consciousness and/or signs of intracranial hypertension. At admission, the clinical-radiological presentation guided the choice of the initial treatment modality: a low GCS score imposed limited imaging, initial resuscitation, and discussion of emergency surgery. In the other patients, the cases were discussed in an interdisciplinary fashion, and in AVMs in which an intranidal or a flow aneurysm could be identified as the likely source of bleeding, endovascular treatment was aimed primarily at obliterating the suspect vascular segment. Conversely, if no source of bleeding was seen on baseline imaging, diagnostic catheter angiography was performed within the first week after onset.

\section{HE Procedure}

Acute surgeries were performed by more than 10 different surgeons over the study period, including junior consultants with limited experience in neurovascular surgery, highlighting the rarity of pediatric ICH. HE was performed using the following general rules. After a large craniotomy was performed under neuronavigation, the cortex was incised where the hematoma was the most superficial, while avoiding eloquent areas. Gentle aspiration of the hematoma along with irrigation with saline aimed for significant brain decompression, but not for complete clot removal so as to avoid AVM rebleeding. Simultaneous targeted resection of the AVM was performed if angioarchitectural characteristics and the surgeons' experience allowed it. If needed, an EVD was placed before or during HE. DC was performed during the initial surgery of $\mathrm{HE}$ in cases of uncontrollable parenchymal swelling and during a second surgical session in cases of refractory intracranial hypertension despite optimal medical treatment according to recommended guidelines. ${ }^{12}$ HEs performed within 24 hours after initial imaging were termed "early," and those performed after 24 hours were called "late."

\section{Statistical Analysis}

Statistical analyses were performed using JMP Pro version 14.0 (SAS Institute Inc., 1989-2019). Continuous variables were expressed as the mean \pm standard deviation. Categorical variables were expressed as absolute number and percentages. We tested the association of clinical and radiological variables with the occurrence of HE, using univariable tests as appropriate according to the variable's nature. Variables associated with HE that had a $\mathrm{p}<0.1$ were entered in a multivariable nominal logistic regression model to test their independent association with 
TABLE 1. Clinical and radiological characteristics of 112 cases of ruptured brain AVMs and comparison between the group with HE and the one without

\begin{tabular}{|c|c|c|c|c|}
\hline Variable & Total, $n=112$ & $\mathrm{HE}, \mathrm{n}=51$ & No HE, $n=61$ & $\mathrm{p}$ Value \\
\hline \multicolumn{5}{|l|}{ Patient characteristics } \\
\hline Age at event, yrs & $9.6 \pm 3.9$ & $9.6 \pm 5.8$ & $9.7 \pm 4.7$ & 0.86 \\
\hline Male sex & $60(54 \%)$ & $25(49 \%)$ & $35(57 \%)$ & 0.78 \\
\hline GCS score & $11.4 \pm 3.9$ & $9.9 \pm 5.6$ & $12.7 \pm 4.4$ & $<0.001$ \\
\hline GCS score $<9$ & $34(30 \%)$ & $23(45 \%)$ & $11(18 \%)$ & 0.002 \\
\hline Neurological deficit & $64(57 \%)$ & $35(69 \%)$ & $29(48 \%)$ & 0.04 \\
\hline Seizures & $83(74 \%)$ & $45(88 \%)$ & $38(62 \%)$ & 0.001 \\
\hline Intracranial hypertension symptoms & $93(83 \%)$ & $46(90 \%)$ & $47(77 \%)$ & 0.08 \\
\hline \multicolumn{5}{|l|}{ Baseline imaging data } \\
\hline $\mathrm{ICH} / \mathrm{TBV}$ & $2.2 \% \pm 1.9 \%$ & $3.2 \% \pm 2.0 \%$ & $1.4 \% \pm 1.8 \%$ & $<0.001$ \\
\hline $\mathrm{ICH}$ vol, ml & $21.8 \pm 15.9$ & $30.7 \pm 19.3$ & $14.4 \pm 17.6$ & $<0.001$ \\
\hline Left-sided ICH & $55(49 \%)$ & $27(53 \%)$ & $28(46 \%)$ & 0.13 \\
\hline Supratentorial ICH & $94(84 \%)$ & $45(88 \%)$ & $49(80 \%)$ & 0.30 \\
\hline Modifed Graeb score & $9.8 \pm 11.4$ & $8.6 \pm 14.4$ & $10.8 \pm 16.3$ & 0.34 \\
\hline Hydrocephalus & $68(61 \%)$ & $31(61 \%)$ & $37(61 \%)$ & $>0.99$ \\
\hline Presence of brain herniation & $49(44 \%)$ & $36(71 \%)$ & $13(21 \%)$ & $<0.001$ \\
\hline Falcine herniation & $28(25 \%)$ & $19(37 \%)$ & $9(15 \%)$ & \multirow{4}{*}{$<0.001$} \\
\hline Temporal herniation & $15(13 \%)$ & $12(24 \%)$ & $3(5 \%)$ & \\
\hline Tonsillar herniation & $6(5 \%)$ & $5(10 \%)$ & $1(2 \%)$ & \\
\hline No herniation & $63(56 \%)$ & $15(29 \%)$ & $48(79 \%)$ & \\
\hline EVD placement & $61(54 \%)$ & $26(51 \%)$ & $35(57 \%)$ & 0.57 \\
\hline \multicolumn{5}{|l|}{ AVM characteristics } \\
\hline Superficial location & $52(46 \%)$ & $32(63 \%)$ & $20(33 \%)$ & 0.002 \\
\hline Any deep AVM drainage & $53(47 \%)$ & $18(35 \%)$ & $35(57 \%)$ & 0.02 \\
\hline Secondary bleeding & $8(7 \%)$ & $3(6 \%)$ & $5(8 \%)$ & 0.73 \\
\hline Presence of AVM-associated aneurysm & $36(32 \%)$ & $15(29 \%)$ & $21(34 \%)$ & 0.55 \\
\hline AVM eloquent cortex & $60(54 \%)$ & $27(53 \%)$ & $33(54 \%)$ & $>0.99$ \\
\hline \multicolumn{5}{|l|}{ Spetzler-Ponce class } \\
\hline A & $72(64 \%)$ & $37(73 \%)$ & $35(57 \%)$ & 0.02 \\
\hline B & $26(23 \%)$ & $12(24 \%)$ & $14(23 \%)$ & \\
\hline C & $14(13 \%)$ & $2(4 \%)$ & $12(20 \%)$ & \\
\hline
\end{tabular}

Values are expressed as absolute number (percentage) or mean $\pm \mathrm{SD}$, as appropriate. Boldface type indicates statistical significance.

HE. Nonsignificant variables were then backward eliminated. Independently of univariable test results, and based on previous knowledge, it was prespecified that the multivariable model for $\mathrm{HE}$ would be adjusted for age, baseline ICH volume, and initial GCS score. We proceeded with the same methodology in order to test associations of clinical-radiological variables and $\mathrm{HE}$ with the clinical outcome. The association between $\mathrm{HE}$ and other surgical procedures was explored using descriptive statistics. Probability curves were calculated for $\mathrm{HE}$ according to initial GCS score and ICH volume as well as ICH/TBV. Two-sided $\mathrm{p}$ values $<0.05$ were considered statistically significant.

\section{Results}

\section{Study Sample and Baseline Characteristics}

A total of 123 children with ICH due to AVM were treated at our institution between 2002 and 2018. Among them, 6 were excluded due to missing clinical data and 13 with isolated IVH. Recurrent bleeding was observed in 8 of 104 children (7.7\%); thus, a total of 112 hemorrhagic events were analyzed. Clinical and radiological findings of the entire study group, including a comparison between the group with and the group without HE, are summarized in Table 1 . In brief, 60 of 112 cases $(53.6 \%)$ were in males and $52(46.4 \%)$ were in females. The mean age at rupture was $9.6 \pm 3.9$ years. The mean initial GCS score at admission was $11.4 \pm 3.9$, intracranial hypertension symptoms were reported in 93 of 112 cases (83\%), a neurological deficit was noted in $64(57.1 \%)$, and seizures in 83 cases $(74.1 \%)$. Most of the ICHs $(84 \%)$ were supratentorial, with a mean ICH volume of $21.8 \pm 15.9 \mathrm{ml}$. Hydrocephalus and brain herniation occurred in $60.7 \%$ and $43.7 \%$ of the cases, respectively. There were 72 Spetzler-Ponce class A (64\%), 


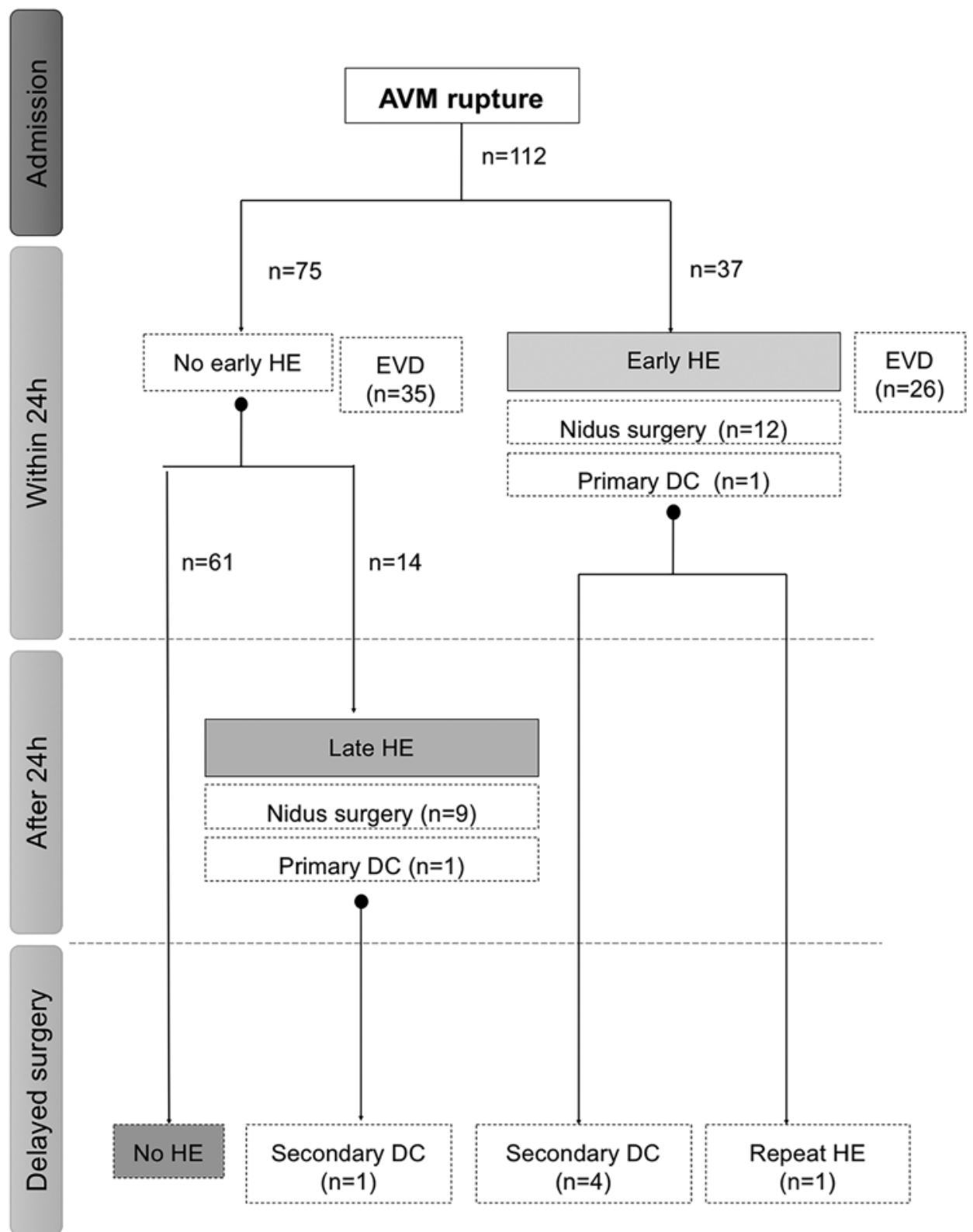

FIG. 1. Chronological flow diagram of primary early HE (within 24 hours after initial cerebral imaging) and late HE (more than 24 hours after initial cerebral imaging), as well as secondary surgical interventions performed in the described cohort.

26 class B (23\%), and 14 class C (13\%) AVMs. The flow diagram in Fig. 1 illustrates the timeline of acute surgical interventions, which will be separately discussed below.

\section{Hemorrhage Evacuation}

In 61 cases (54.5\%), children did not undergo acute surgical management of the ICH. HE was performed in 51 cases $(45.5 \%)$, of which 37 cases $(73 \%)$ were performed early. Due to secondary neurological deterioration, late HE was necessary in 14 cases (mean time interval to initial cerebral imaging 3.3 days, range $1-12$ days). In 7 cases, catheter angiography was performed before HE, including 2 cases with presurgical embolization. In 2 cases, concur- rent DC was required because of uncontrolled brain swelling. There was a significant AVM rupture during HE in 1 case that could eventually be controlled after extensive AVM coagulation.

In univariate analysis, patients with HE had a lower initial GCS score than patients without HE (9.9 vs 12.7, p < $0.001)$, more often had neurological deficits $(69 \%$ vs $48 \%$, $\mathrm{p}=0.04)$, more commonly had seizures $(88 \%$ vs $62 \%$, $\mathrm{p}$ $=0.001)$, had more frequent brain herniation on initial cerebral imaging ( $73 \%$ vs $27 \%$, p < 0.001), had larger ICH volumes $(30.7 \pm 19.3 \mathrm{ml}$ vs $14.4 \pm 17.6 \mathrm{ml}, \mathrm{p}<0.001)$, and had higher ICH/TBV ratios $(3.2 \% \pm 2.0 \%$ vs $1.4 \% \pm 1.8 \%$, $\mathrm{p}<0.001$ ). The underlying AVM was more frequently superficial (63\% vs 33\%), with lower Spetzler-Ponce class 
TABLE 2. Multivariable model analysis of predictive factors for HE after ruptured brain AVM

\begin{tabular}{|c|c|c|c|c|c|}
\hline \multicolumn{3}{|c|}{ Model 1} & \multicolumn{3}{|c|}{ Model 2} \\
\hline Variable & aOR $(95 \% \mathrm{Cl})$ & $p$ Value & Variable & aOR $(95 \% \mathrm{Cl})$ & $\mathrm{p}$ Value \\
\hline GCS score, per point increase & $0.83(0.71-0.97)$ & $<0.001$ & GCS score, per point increase & $0.84(0.71-0.99)$ & 0.04 \\
\hline Age at event, per yr increase & $0.97(0.85-1.11)$ & 0.67 & Age at event, per yr increase & $0.99(0.86-1.13)$ & 0.86 \\
\hline $\mathrm{ICH} / \mathrm{TBV}$, per \% increase & $18.6(13-26.5)$ & 0.01 & ICH/TBV, per \% increase & $21.2(14.2-31.8)$ & $<0.001$ \\
\hline Superficial AVM & $3.75(1.25-11.31)$ & 0.02 & Superficial AVM & $3.57(1.14-11.22)$ & 0.03 \\
\hline \multirow[t]{3}{*}{ Presence of brain herniation } & $3.73(1.34-10.39)$ & $<0.001$ & Falcine herniation* & $2.04(0.61-6.79)$ & 0.24 \\
\hline & & & Temporal herniation* & $7.71(1.34-44.32)$ & 0.02 \\
\hline & & & Tonsillar herniation* & $14.3(1.28-159.98)$ & 0.001 \\
\hline
\end{tabular}

Boldface type indicates statistical significance.

* "No herniation" used as reference.

(97\% class A vs $70 \%$ class B) (Table 1). In the fully adjusted multivariable model, factors independently associated with HE included high ICH/TBV (adjusted odds ratio [aOR] 18.6, 95\% CI 13.0-26.5 for each percent increase; $\mathrm{p}=0.01$ ), low initial GCS score, presence of brain herniation, and superficial AVM location (aOR 3.75, 95\% CI 1.25-11.3; $\mathrm{p}=0.02$ ) (Table 2). When considering the subtype of brain herniation, tonsillar herniation was most strongly associated with $\mathrm{HE}$ (aOR 14.3, 95\% CI 1.28159.98; $\mathrm{p}=0.001)$. Figure 2 is a graphical representation of the adjusted multivariable analysis (model 1 in Table 2) probability of HE plotted against initial GCS score and ICH volume.

Compared with the group undergoing late HE $(n=14$ cases), the group with early HE ( $\mathrm{n}=37$ cases) had lower initial GCS scores $(8.6 \pm 0.6$ vs $13.2 \pm 0.9, \mathrm{p}<0.001)$, more frequent altered consciousness (59\% vs 7\%, p < 0.001), a more infratentorial location $(16.2 \%$ vs $0 \%, \mathrm{p}=0.002)$, and a more frequent presence of brain herniation on initial cerebral imaging $(81.1 \%$ vs $42.9 \%, \mathrm{p}=0.01)$. Conversely, no significant difference of ICH/TBV was found $(3.2 \% \pm$ $1.4 \%$ vs $2.9 \% \pm 1.4 \%, \mathrm{p}=0.79)$. Predictive factors for the early HE timing included low initial GCS score, infratentorial AVM, and the presence of brain herniation on initial cerebral imaging.

\section{AVM Nidal Resection at the Acute Phase}

Twenty-one of 51 patients (41\%) had concurrent AVM nidus resection and HE (12 during early HE and 9 during late HE) (Fig. 3). Resected AVMs were either SpetzlerPonce class A (7 Spetzler-Martin grade I and 11 SpetzlerMartin grade II) or B (3 Spetzler-Martin grade III). Hence, nidal surgery was performed in $69 \%$ of Spetzler-Ponce class A AVMs and 25\% of Spetzler-Ponce class B AVMs undergoing HE. Factors associated with concurrent nidus resection, compared with exclusive HE, were higher initial GCS scores (mean $12.95 \pm 2.5$ vs $8.6 \pm 3.7, p<0.001$ ) and

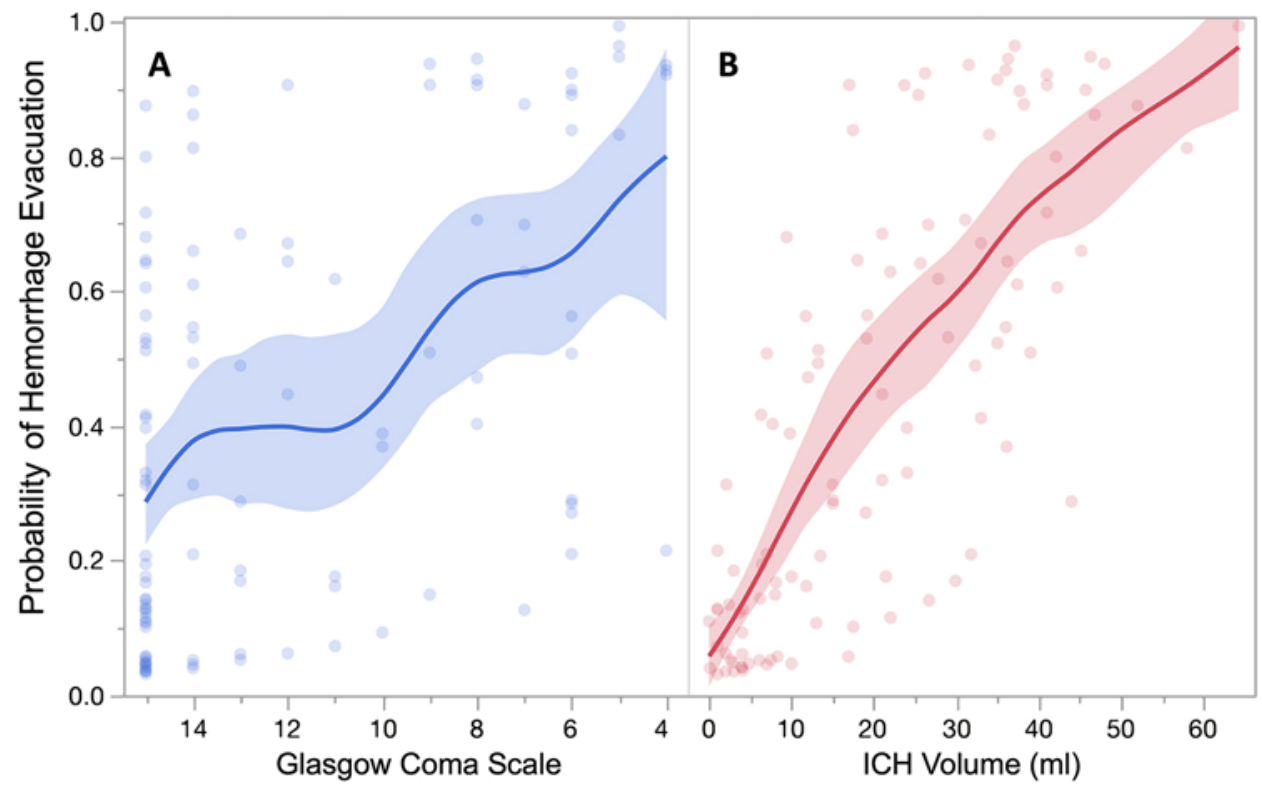

FIG. 2. Probability of HE in the fully adjusted model, plotted according to the initial GCS score (A) and ICH volume (B). Figure is available in color online only. 
S M Grade I-II

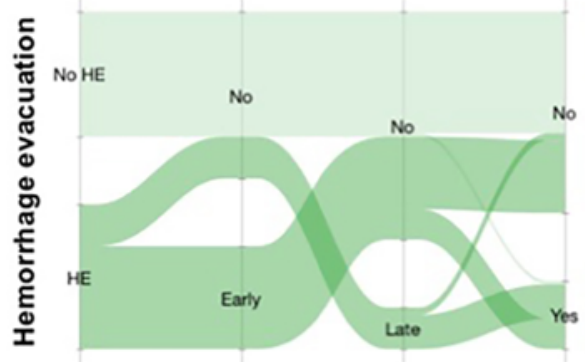

Early HE Late HE Nidal surgery
S M Grade III

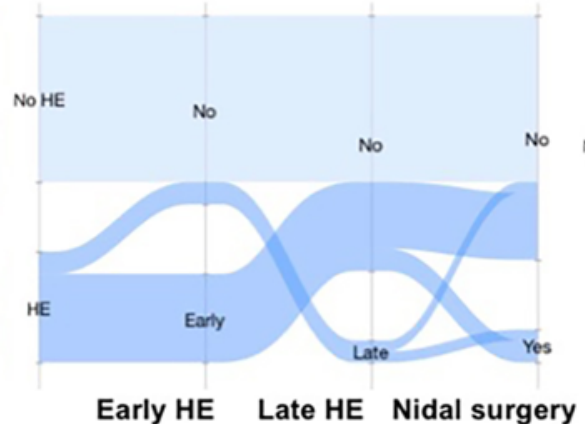

S M Grade V-IV

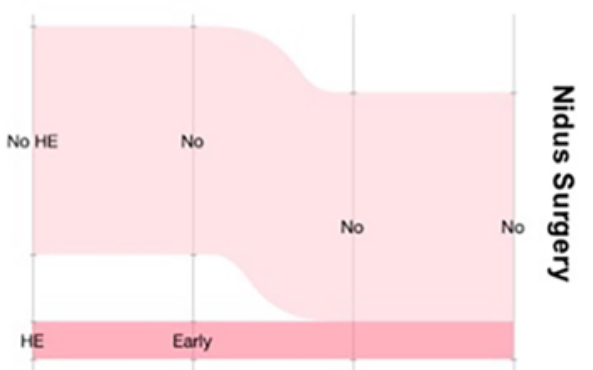

Early HE Late HE Nidal surgery

FIG. 3. Sankey flow diagram of surgical interventions. Illustration of the chronology of surgical interventions according to the Spetzler-Ponce grade of the ruptured AVMs: Spetzler-Ponce class A (left, Spetzler-Martin grade I-II), class B (center, SpetzlerMartin grade III), and class C (right, Spetzler-Martin grade IV-V). The widths of the bands represent the proportion of cases per group undergoing early or late surgery as well as targeted nidus resection. S M Grade = Spetzler-Martin grade. Figure is available in color online only.

accordingly less frequent altered consciousness (5\% vs $63.6 \%, \mathrm{p}<0.001)$, more frequent superficial AVMs $(92.3 \%$ vs $52.6 \%, \mathrm{p}=0.02)$, and a late $\mathrm{HE}(47.6 \%$ vs $8 \%, \mathrm{p}=0.03)$. There was a tendency toward lower Spetzler-Ponce classes in the AVM resection group $(85.7 \%$ Spetzler-Ponce class A vs $65.3 \%, \mathrm{p}=0.07)$. Complete resection was attempted in 9 of 21 cases $(43 \%)$. Five underwent diagnostic catheter angiography before surgery, and angioarchitectural characteristics were evaluated with injected CT scans in the remainder.

\section{Surgery for Secondary Intracranial Hypertension}

Five patients underwent secondary decompressive craniectomy because of refractory intracranial hypertension despite optimal medical treatment, with a mean delay of $3.5 \pm 4.4$ days after admission. These patients had lower initial GCS scores $(7.1 \pm 3.7$ vs $11.8 \pm 3.7, \mathrm{p}=0.02)$, larger ICH volumes $(39.0 \pm 16.7 \mathrm{ml}$ vs $20.5 \pm 15.4 \mathrm{ml}, \mathrm{p}=0.02)$, a larger ICH/TBV $(4.1 \% \pm 2.0 \%$ vs $2.1 \% \pm 1.6 \%, \mathrm{p}=0.02)$, and more frequent brain herniation on initial cerebral imaging than patients who did not require DC.

\section{Outcome}

During the acute phase, rebleeding occurred in 2 cases, after a delay of 2 and 7 days since admission for the first bleeding episode. One of the patients, with a Spetzler-Martin grade II AVM, had undergone early HE with concurrent partial AVM resection and underwent successful reoperation at day 7; the other, with a Spetzler-Martin grade IV AVM, died after 48 hours.

Postoperative complications requiring additional surgery were encountered in 2 cases. One patient underwent reoperation because of wound healing difficulties, and the other patient required enlargement of the craniectomy due to recurrent intracranial hypertension.

At 18 months, the clinical outcome was available for 101 of 104 patients, and it was pending for the 3 remaining children. Distribution according to the KOSCHI scale was as follows for these 101 patients: KOSCHI 5B in 21 cases $(20.8 \%)$; KOSCHI $5 \mathrm{~A}$ in 38 (37.6\%); KOSCHI 4B in $23(22.8 \%)$; KOSCHI $4 \mathrm{~A}$ in $6(5.9 \%)$; KOSCHI 3 in 8
(7.9\%); KOSCHI 2 in 1 (1\%); and 4 patients (4\%) died. In summary, $58.4 \%$ of the patients had a favorable outcome (KOSCHI 5A and 5B); $28.7 \%$ had an unfavorable outcome with mild disabilities yet functioning independently (KOSCHI 4A and 4B); $8.9 \%$ had severe disabilities or were in a vegetative state (KOSCHI 3 and 2); and $4 \%$ died. Hence, $87 \%$ were functioning independently 18 months after the bleeding event.

There was no significant difference between patients with and those without $\mathrm{HE}$ concerning favorable outcome (KOSCHI $<5 \mathrm{~A}, 52 \%$ vs $33 \%$; $\mathrm{p}=0.05)$ and severe disability (KOSCHI < 4A, $18 \%$ vs $8 \%$; $\mathrm{p}=0.16$ ). There was no significant difference either between patients undergoing early $\mathrm{HE}$ compared to those with late $\mathrm{HE}$ (KOSCHI $<5 \mathrm{~A}, 60 \%$ vs $33 \%$, respectively; $\mathrm{p}=0.07$ ) or between patients with late $\mathrm{HE}$ compared to those with no HE required (KOSCHI $<5 \mathrm{~A}, 33 \%$ vs $31 \%$, respectively; $\mathrm{p}=0.13$ ).

\section{Discussion}

Compiling more than 110 pediatric ruptured brain AVM cases, from the hyperacute phase to 18 months after stroke onset, we showed that a surgical strategy comprising $\mathrm{HE}$ in nearly half of children is associated with functional independence in approximately $87 \%$ and a mortality rate as low as $3.8 \%$. Interestingly, outcome was comparable between surgically and nonsurgically treated patients despite significantly different initial clinical presentation. In our opinion, these results reinforce an interventionist surgical strategy, based on the severity of initial clinical status as well as baseline imaging, including brain herniation and large $\mathrm{ICH}$ volumes.

Previous reports on emergency surgery in children with ruptured AVMs are scarce. In Nerva et al., ${ }^{13}$ between 2005 and 2012, 16 of 27 patients (59\%) underwent nonelectivethus emergency or urgent-surgery after a mean delay of 4 days between admission and surgery. This included 3 cases of primary DC and 2 cases of secondary DC due to postoperative cerebral edema. ${ }^{13}$ Shtaya et al. reported, over a period of more than 20 years (1994-2016), HE in 5 of 37 children (14\%) during the initial hospitalization for ruptured brain AVM. ${ }^{14}$ Singhal et al. described HE in 12 
of 15 children (80\%) presenting with a ruptured AVM and an initial GCS score of $<9$, including nidus resection in 9 cases, with a median 4-hour interval after admission and a mortality rate of $20 \% .^{15}$

When comparing our results to adult cohorts of patients with ruptured AVM, the most striking difference is the higher frequency of HE, as well as the shorter time interval between initial cerebral imaging and surgery. ${ }^{16,17}$ Beecher et al. described emergency surgery in only 6 of 163 cases with ruptured AVM. ${ }^{18}$ In another adult cohort of 59 patients from Finland, the median delay for surgery was 2 days after onset of symptoms. ${ }^{19}$ Both differences are striking because the mean ICH volume was not higher in the present study than in adults when taking into account whole-brain volume (mean ICH/TBV 2.2\%). Indeed, $60 \mathrm{ml}$ is frequently used as a cutoff value for surgery in adults and is comparable to a mean ICH/TBV of $4 \%$ in a child. Rapid decompensation of intracranial hypertension in children may be explained by differences in brain parenchyma compliance, ${ }^{20}$ fewer CSF-related compensatory mechanisms, and a higher rate of seizures. Altogether, despite the fact that the present study may reflect a single institution's habits, the large proportion of early HE in this large sample strongly supports the immediate transfer of children after ICH diagnosis to a tertiary care center with a pediatric neurointensive care unit and neurosurgical facilities.

Identifying determinants of surgical HE is important, because these emergency procedures are often performed in the off-hours by surgeons with various levels of neurovascular experience. ${ }^{19}$ Yet, few data exist on these specific procedures, and there are no evidence-based recommendations, because both children and underlying vascular lesions were excluded from randomized controlled trials investigating the benefits of HE (such as Surgical Trial in Intracerebral Hemorrhage [STICH] I or II) ${ }^{21,22}$ Experts advocate ICH evacuation in cases of "large, life-threatening hematoma" 23 or in cases of "decreased level of consciousness due to an ICH" and for "hematomas larger than $30 \mathrm{ml}$ in the temporal lobe or cerebellum or $60 \mathrm{ml}$ in other parts of the hemispheres." ${ }^{24}$ In the present study, HE was more frequently performed in children with altered consciousness, larger hematoma, or radiological signs of herniation, which emphasizes the need for a global clinical and imaging evaluation for the surgical indication. Although the sample size of our cohort did not allow us to derive and validate a clinical-radiological score to guide surgical indications, we supply probability curves, which may help in decision-making, notably for children with initial GCS scores $>10$ and intermediate ICH volumes. As a representative example, in a 10-year-old child with an initial GCS score of 11 and a superficial ruptured AVM, the probabilities of evacuating the ICH with ICH/TBV rates of $0.5 \%$, $2 \%$, and $5 \%$ would be $25.9 \%, 46 \%$, and $84 \%$, respectively.

Whereas there is no ambiguity regarding the indication for emergency surgery for a child who presents in extremis, the timing of nidal surgical treatment for ruptured AVMs has been a controversial topic. In the absence of a recommended timeline, timing is a subject of significant variation in clinical practice, between AVM resection concurrent with lifesaving emergency craniotomy and clot removal within a few hours after onset versus delayed for weeks..$^{18}$ Interestingly, in the present study, 21 of 51 patients $(41 \%)$ had concurrent HE and AVM nidus resection, in line with previous series reporting from $20 \%{ }^{14}$ to $84 \%^{22}$ early AVM removal. The attitude in favor of delayed nidus resection originated from concerns about compression of the nidus by the hematoma, hindering the visualization of the AVM architecture and potentially damaging the brain tissue. Therefore, early AVM surgery can increase both the risk of an incomplete removal ${ }^{24}$ and the risk of neurological deficit. ${ }^{23,25}$ The fact that AVMs are unlikely to rebleed very early has also been used to support this opinion. However, from another neurosurgical perspective, fresh AVM-related hematoma may facilitate resection, creating a cavity and offering a good cleavage plane. This could explain why in the present series AVM resection was more frequently performed after 24 hours in patients with a higher GCS score and more superficial AVM location.

In addition, immediate resection prevents a new hemorrhage and facilitates the rehabilitation of the patient. Hence, some authors advocate concurrent HE and microsurgical resection in a single procedure at the acute phase, notably for low Spetzler-Martin grade AVM, with comparable outcomes to delayed surgery and a reduction of the rebleeding risk..$^{16,19,26}$ However, in the present study we did not observe an increased risk of rebleeding in children with delayed AVM excision and did not find such reports in the pediatric literature. We encountered a rebleeding rate of $7.7 \%$, with only 2 cases of early rebleeding during initial hospitalization. This rate is comparable to the rebleeding rate of 6\%-15.6\% within the first year after AVM rupture reported in adult series. ${ }^{27}$ Combined endovascular and microsurgical procedures with preoperative embolization is proposed even at the acute stage because it increases the obliteration rate of AVM even for grade III lesions and decreases intraoperative risk. ${ }^{28,29}$ Yet, this solution may not appear ideal for children with ICH and severe clinical presentation (e.g., coma, third nerve palsy) who require emergency surgery without the time for extensive imaging ${ }^{30}$ or neurointerventional treatment.

It is important to note that, despite the high rate of emergency neurosurgical procedures in our sample, the overall mortality rate $(3.8 \%)$ was low, and lower than in previous reports in this age group after AVM rupture (ranging between 5\% and 20\%). ${ }^{13,31-33}$ Functional independence was observed in almost 9 in 10 children at 18 months. Previous studies reported similar independent functioning in $83 \%-92 \%$ and favorable outcome in $61 \%-84 \%$ of surgically treated ruptured AVMs in children, but with shorter-term follow-up. ${ }^{13,15,34}$ Long-term outcomes for children with early $\mathrm{HE}$ were comparable to those with delayed $\mathrm{HE}$, and similar between children with delayed HE and those without HE. This is despite the fact that the patients with life-threatening hematomas, and therefore disproportionately more severe initial presentations, were naturally treated with minimal delays. These results reinforce the role of acute neurosurgical decompressive approaches in determining clinical outcome.

\section{Study Strengths and Limitations}

Our study has several strengths, including a large cohort 
of consecutively treated children, long-term follow-up, and use of a multivariate model aiming to enable the analysis of the time interval between ICH onset and surgery as a potential predicting factor with regard to outcome. Limitations included the retrospective design and the inclusion of patients with infratentorial AVMs, because infratentorial ICH often necessitates immediate surgery, and timing of treatment is less controversial. Also, one may argue that too many surgeons with various experience in pediatric neurovascular surgery were involved, which may have maximized surgeon experience as a confounding factor. However, in our opinion, even though AVM neurosurgical management is a difficult task, our study offers real-world care conditions with generalizable results. Prospective investigations involving a larger number of centers are required to validate the findings of this study.

\section{Conclusions}

The HE rate in children with ruptured brain AVM is high and is associated with clinical and imaging baseline severity. With almost half of children requiring HE in this study, the mortality rate was limited to less than $4 \%$, supporting an intensive approach for children with severe presentations. In addition, neither the time interval between AVM bleeding and HE nor the HE itself negatively influenced clinical outcome, with functional independence observed in 9 of 10 children at 18 months. The advantage of concurrent acute nidal resection is balanced between the decreased risk of early rebleeding and the elevated risk of increased cerebral damage.

\section{Acknowledgments}

We thank the Imagine Institute, and notably Dr. N. Garcelon, for help with the Dr. Warehouse software.

\section{References}

1. Ding D, Starke RM, Kano H, et al. International multicenter cohort study of pediatric brain arteriovenous malformations. Part 1: Predictors of hemorrhagic presentation. J Neurosurg Pediatr. 2017;19(2):127-135.

2. Guédon A, Blauwblomme T, Boulouis G, et al. Predictors of outcome in patients with pediatric intracerebral hemorrhage: development and validation of a modified score. Radiology. 2018;286(2):651-658.

3. Blauwblomme T, Bourgeois M, Meyer P, et al. Long-term outcome of 106 consecutive pediatric ruptured brain arteriovenous malformations after combined treatment. Stroke. 2014;45(6):1664-1671.

4. Boulouis G, Blauwblomme T, Hak JF, et al. Nontraumatic pediatric intracerebral hemorrhage. Stroke. 2019;50(12): 3654-3661.

5. Gross BA, Lai PMR, Du R. Hydrocephalus after arteriovenous malformation rupture. Neurosurg Focus. 2013;34(5): E11.

6. von Elm E, Altman DG, Egger M, et al. The Strengthening the Reporting of Observational Studies in Epidemiology (STROBE) statement: guidelines for reporting observational studies. Lancet. 2007;370(9596):1453-1457.

7. Garcelon N, Neuraz A, Salomon R, et al. A clinician friendly data warehouse oriented toward narrative reports: Dr. Warehouse. J Biomed Inform. 2018;80:52-63.

8. Calvert S, Miller HE, Curran A, et al. The King's Outcome Scale for Childhood Head Injury and injury severity and out- come measures in children with traumatic brain injury. Dev Med Child Neurol. 2008;50(6):426-431.

9. Stricker S, Boulouis G, Benichi S, et al. Hydrocephalus in children with ruptured cerebral arteriovenous malformation. J Neurosurg Pediatr. 2020;26(3):283-287.

10. Morgan TC, Dawson J, Spengler D, et al. The Modified Graeb Score: an enhanced tool for intraventricular hemorrhage measurement and prediction of functional outcome. Stroke. 2013;44(3):635-641.

11. Spetzler RF, Ponce FA. A 3-tier classification of cerebral arteriovenous malformations. J Neurosurg. 2011;114(3): 842-849.

12. American Association for the Surgery of Trauma, Child Neurology Society, International Society for Pediatric Neurosurgery, et al. Guidelines for the acute medical management of severe traumatic brain injury in infants, children, and adolescents. J Trauma. 2003;54(6 Suppl):S235-S310.

13. Nerva JD, Kim LJ, Barber J, et al. Outcomes of multimodality therapy in pediatric patients with ruptured and unruptured brain arteriovenous malformations. Neurosurgery. 2016; 78(5):695-707.

14. Shtaya A, Millar J, Sparrow O. Multimodality management and outcomes of brain arterio-venous malformations (AVMs) in children: personal experience and review of the literature, with specific emphasis on age at first AVM bleed. Childs Nerv Syst. 2017;33(4):573-581.

15. Singhal A, Adirim T, Cochrane D, Steinbok P. Pediatric patients with poor neurological status and arteriovenous malformation hemorrhage: an outcome analysis. J Neurosurg Pediatr. 2011;7(5):462-467.

16. Jafar JJ, Rezai AR. Acute surgical management of intracranial arteriovenous malformations. Neurosurgery. 1994;34(1): $8-13$.

17. Westphal M, Cristante L, Grzyska U, et al. Treatment of cerebral arteriovenous malformations by neuroradiological intervention and surgical resection. Acta Neurochir (Wien). 1994;130(1-4):20-27.

18. Beecher JS, Lyon K, Ban VS, et al. Delayed treatment of ruptured brain AVMs: Is it ok to wait? J Neurosurg. 2018;128(4): 999-1005.

19. Hafez A, Oulasvirta E, Koroknay-Pál P, et al. Timing of surgery for ruptured supratentorial arteriovenous malformations. Acta Neurochir (Wien). 2017;159(11):2103-2112.

20. Kiening KL, Schoening W, Unterberg AW, et al. Assessment of the relationship between age and continuous intracranial compliance. In: Poon WS, Avezaat CJJ, Chan M, et al, eds. Intracranial Pressure and Brain Monitoring XII. Vol 95. Springer-Verlag Wien; 2005:293-297.

21. Mendelow AD, Gregson BA, Rowan EN, et al. Early surgery versus initial conservative treatment in patients with spontaneous supratentorial lobar intracerebral haematomas (STICH II): a randomised trial. Lancet. 2013;382(9890):397-408.

22. Mendelow AD, Gregson BA, Fernandes HM, et al. Early surgery versus initial conservative treatment in patients with spontaneous supratentorial intracerebral haematomas in the International Surgical Trial in Intracerebral Haemorrhage (STICH): a randomised trial. Lancet. 2005;365(9457): 387-397.

23. Ogilvy CS, Stieg PE, Awad I, et al. AHA Scientific Statement: Recommendations for the management of intracranial arteriovenous malformations: a statement for healthcare professionals from a special writing group of the Stroke Council, American Stroke Association. Stroke. 2001;32(6):1458-1471.

24. Martinez JL, Macdonald RL. Surgical strategies for acutely ruptured arteriovenous malformations. Front Neurol Neurosci. 2015;37:166-181.

25. Zacharia BE, Vaughan KA, Jacoby A, et al. Management of ruptured brain arteriovenous malformations. Curr Atheroscler Rep. 2012;14(4):335-342. 
26. Pavesi G, Rustemi O, Berlucchi S, et al. Acute surgical removal of low-grade (Spetzler-Martin I-II) bleeding arteriovenous malformations. Surg Neurol. 2009;72(6):662-667.

27. Can A, Gross BA, Du R. The natural history of cerebral arteriovenous malformations. Handb Clin Neurol. 2017;143: $15-24$.

28. Alias Q, Boulouis G, Blauwblomme T, et al. First line Onyx embolization in ruptured pediatric arteriovenous malformations: safety and efficacy. Clin Neuroradiol. 2019.

29. Gross BA, Storey A, Orbach DB, et al. Microsurgical treatment of arteriovenous malformations in pediatric patients: the Boston Children's Hospital experience. J Neurosurg Pediatr. 2015;15(1):71-77.

30. Meyer PG, Orliaguet GA, Zerah M, et al. Emergency management of deeply comatose children with acute rupture of cerebral arteriovenous malformations. Can J Anaesth. 2000; 47(8):758-766.

31. Humphreys RP, Hoffman HJ, Drake JM, Rutka JT. Choices in the 1990s for the management of pediatric cerebral arteriovenous malformations. Pediatr Neurosurg. 1996;25(6): 277-285.

32. Kiriş T, Sencer A, Sahinbaş M, et al. Surgical results in pediatric Spetzler-Martin grades I-III intracranial arteriovenous malformations. Childs Nerv Syst. 2005;21(1):69-76.

33. Kondziolka D, Humphreys RP, Hoffman HJ, et al. Arteriovenous malformations of the brain in children: a forty year experience. Can J Neurol Sci. 1992;19(1):40-45.

34. Sanchez-Mejia RO, Chennupati SK, Gupta N, et al. Superior outcomes in children compared with adults after microsurgical resection of brain arteriovenous malformations. J Neurosurg. 2006;105(2 Suppl):82-87.

\section{Disclosures}

The authors report no conflict of interest concerning the materials or methods used in this study or the findings specified in this paper.

\section{Author Contributions}

Conception and design: Blauwblomme, Stricker, Boulouis, Benichi, Naggara. Acquisition of data: Blauwblomme, Stricker, Boulouis, Benichi, Gariel, Garzelli, Hak, Alias, Kerleroux, Beccaria, Chivet, de Saint Denis, James, Paternoster, Zerah, Kossorotoff, Boddaert, Brunelle, Meyer, Puget, Naggara. Analysis and interpretation of data: Blauwblomme, Stricker, Boulouis, Benichi, Bourgeois, Naggara. Drafting the article: Blauwblomme, Stricker, Boulouis, Naggara. Critically revising the article:

Blauwblomme, Stricker, Boulouis, Benichi, Naggara. Reviewed submitted version of manuscript: Blauwblomme, Stricker,

Boulouis, Benichi, Naggara. Approved the final version of the manuscript on behalf of all authors: Blauwblomme. Statistical analysis: Stricker, Boulouis. Administrative/technical/material support: Stricker, Boulouis. Study supervision: Blauwblomme, Benichi, Naggara.

\section{Correspondence}

Thomas Blauwblomme: Hôpital Necker, Paris, France. thomas.blauwblomme@aphp.fr. 